\title{
Towards a Faddeev-AGS description of $(d, p)$ reactions with heavy nuclei: Regularizing integrals with Coulomb functions.
}

\author{
V. Eremenko, ${ }^{1,5, a}$, L. Hlophe ${ }^{1}$, Ch. Elster ${ }^{1, b}$, F. M. Nunes ${ }^{2}$, I. J. Thompson ${ }^{3}$, G. Arbanas ${ }^{4}$, and \\ J. E. Escher ${ }^{3}$
}

TORUS Collaboration ${ }^{\dagger}$ (http://reactiontheory.org)

${ }^{1}$ INPP and Department of Physics and Astronomy, Ohio University, Athens, Ohio 45701, USA
${ }^{2}$ NSCL and Dept. of Physics and Astronomy, Michigan State University, East Lansing, Michigan 48824,
${ }^{3}$ Lawrence Livermore National Laboratory, L-414, Livermore, California 94551, USA
${ }^{4}$ Reactor and Nuclear Systems Division, Oak Ridge National Laboratory, Oak Ridge, TN 37831, USA
${ }^{5}$ SINP M.V. Lomonosov Moscow State University, Moscow, 119991, Russia

\begin{abstract}
The repulsive Coulomb force poses severe challenges when describing $(d, p)$ reactions for highly charged nuclei as a three-body problem. Casting Faddeev-AGS equations in a Coulomb basis avoids introducing screening of the Coulomb force. However, momentum space partial-wave $t$-matrix elements need to be evaluated in this basis. When those $t$-matrices are separable, the evaluation requires the folding of a form factor, depending on one momentum variable, with a momentum space partial-wave Coulomb function, which has a singular behavior at the external momentum $q$. We developed an improved regularization scheme to calculate Coulomb distorted form factors as the integral over the Coulomb function and complex nuclear form factors.
\end{abstract}

\section{Introduction}

Direct reactions involving rare isotopes offer an important tool for understanding the structure of such nuclei. Deuteron induced reactions are attractive from the experimental perspective, since deuterated targets are readily available. Theoretically they are attractive, since the scattering problem can be reduced to an effective three-body problem, which can be solved exactly using Faddeev techniques. The momentum space Faddeev equations (here in the Alt-Grassberger-Sandhas form) have successfully been solved for $(d, p)$ reactions involving light nuclei [1]. However, the screening technique employed to handle the Coulomb force encounters technical difficulties, when applied to $(d, p)$ reactions with heavier nuclei [2]. An alternative to the screening procedure is a solution of the Faddeev-AGS equations in the Coulomb basis. This was suggested and carried out in Ref. [3] using real two-body transition operators in separable form, neglecting spin degrees of freedom.

Casting Faddeev-AGS equations in the Coulomb basis requires the evaluation of momentum-space partial-wave matrix elements of two-body transition operators,

$$
t_{l}^{C}\left(q^{\prime}, q, E\right)=\int d p^{\prime} d p \psi_{l, q^{\prime}, \eta^{\prime}}^{C}\left(p^{\prime}\right)^{\dagger} t_{l}\left(p^{\prime}, p, E\right) \psi_{l, q, \eta}^{C}(p) .
$$

\footnotetext{
ae-mail: to@vsl.name

be-mail: elster@ohio.edu
} 
If $t_{l}(E)$ is separable, one needs to consider

$$
t_{l}^{C}\left(q, q^{\prime}, E\right)=\sum_{z y} u_{l, z}^{C}(q) \lambda_{l, z y}(E) u_{l, y}^{C}\left(q^{\prime}\right)^{\dagger} .
$$

In this case only the folding of a form factor $u_{l}(p)$, depending on one momentum variable, with partial-wave Coulomb function $\psi_{l, q}^{C}(p)$, for which we developed the numerical procedures in Ref. [6], is required:

$$
u_{l, z}^{C}(q)=\int \frac{d p p^{2}}{2 \pi^{2}} u_{l, z}(p) \psi_{l, q, \eta}^{C}(p)^{*}, \quad \text { and } \quad u_{l, y}^{C}\left(q^{\prime}\right)^{\dagger}=\int \frac{d p p^{2}}{2 \pi^{2}} u_{l, y}(p) \psi_{l, q^{\prime}, \eta}^{C}(p) .
$$

Both integrals have an oscillatory singularity in the point $p=q$, where $q$ is the external momentum. The partial-wave Coulomb functions are given by

$$
\psi_{l, q, \eta}^{C}(p) \stackrel{p \rightarrow q}{=} \psi_{l, q, \eta}^{C}(\chi) \stackrel{\chi \rightarrow 0}{=} \mathcal{A}(q, l, \eta)\left[\frac{\mathcal{B}(\chi, q, l, \eta)}{(\chi+i 0)^{1+i \eta}}-\frac{\mathcal{B}(\chi, q, l, \eta)^{*}}{(\chi-i 0)^{1-i \eta}}\right], \quad \chi \equiv p-q .
$$

To evaluate $u_{l, z}^{C}(q)$, one needs to regularize the integral in Eq. (3). In Ref. [3] this regularization is performed by using a Gel'fand-Shilov (see Ref. [4]) technique for real form factors. When working with form factors describing complex nucleon-nucleus potentials, the regularization scheme must be constructed for complex form factors.

\section{Regularization Procedure}

Here we present a regularization scheme for calculating Coulomb distorted form factors as integrals over a Coulomb function and a complex form factor, which differs from the scheme presented in Ref. [5]. The key point of the work of Ref. [5] is that the integral is regularized only in a tiny neighborhood $\left(\Delta \sim 10^{-6} \mathrm{fm}^{-1}\right)$ around $q$. That allows that the regularized singular part of the folding integral can be reduced to an analytic expression. However, the non-regularized parts of the integral must be calculated quite close to the value of $q$, which requires a lot of integration mesh points independent of the numerical integration quadrature used. For example, we used thousands of GaussLegendre quadrature points to obtain results converged to 3 significant figures.

To avoid using an excessive number of integration points, we developed here the new version of the regularization scheme, where we apply the regularization in a small, but finite neighborhood $\left(\Delta \sim 10^{-3} \mathrm{fm}^{-1}\right)$ around $q$. Now the non-regularized integrals are converging much faster to an even better accuracy (see Fig. 1), while the regularized part is still mostly dominated by the analytical terms. Despite the fact that we need to calculate the regularized integral, we do not need to calculate it as accurately, as we had to calculate the non-regularized parts in Ref. [5]. Thus, the required amount of CPU time is greatly reduced.

Similar to Ref. [5], we start by splitting the folding integral into regularized and non-regularized parts,

$$
u_{l, y}^{C}\left(q^{\prime}\right)^{\dagger}=I_{0}^{q^{\prime}-\Delta}+I_{q^{\prime}-\Delta}^{q^{\prime}+\Delta}+I_{q^{\prime}+\Delta}^{\infty}, \quad I_{a}^{b}=\int_{a}^{b} \frac{d p p^{2}}{2 \pi^{2}} u_{l, y}(p) \psi_{l, q^{\prime}, \eta}^{C}(p) .
$$

We first focus on the regularized part $I_{q-\Delta}^{q+\Delta}$, which we rewrite by substituting Eq. (4) into Eq. (5) as

$$
I_{q^{\prime}-\Delta}^{q^{\prime}+\Delta} \equiv \mathcal{I}_{-\Delta}^{\Delta}=\frac{\mathcal{A}\left(q^{\prime}, l, \eta\right)}{2 \pi^{2}}\left(\mathcal{I}_{+}-\mathcal{I}_{-}\right), \quad \quad \mathcal{I}_{ \pm} \equiv \int_{-\Delta}^{\Delta} d \chi \frac{\phi_{ \pm}\left(\chi, q^{\prime}, l, \eta\right)}{(\chi \pm i 0)^{1 \pm i \eta}}
$$




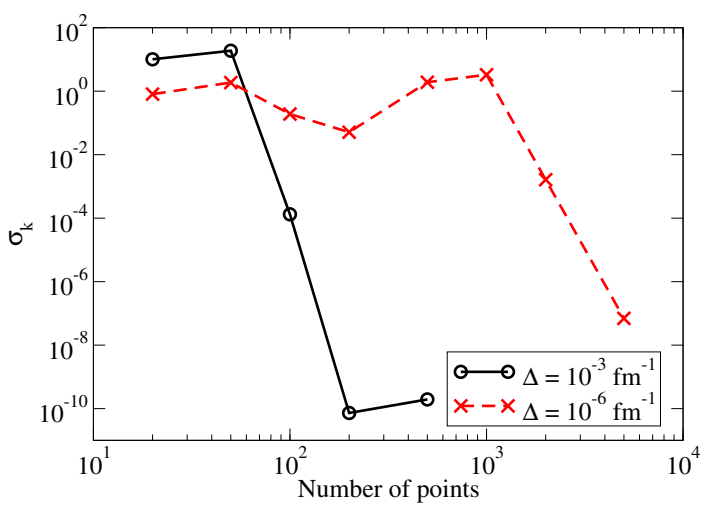

Figure 1. The error plot of the non-regularized integrals $I_{a}^{b}$ with the same lower limit $a=0.4 \mathrm{fm}^{-1}$, but different upper limits: [solid black] $b=0.499 \mathrm{fm}^{-1}$ (i.e. $\Delta=1 \cdot 10^{-3} \mathrm{fm}^{-1}$ ), [dashed red] $b=0.499999 \mathrm{fm}^{-1}\left(\Delta=1 \cdot 10^{-6} \mathrm{fm}^{-1}\right)$. The parameters are: $q=0.5 \mathrm{fm}^{-1}, l=0, \eta=5.3$. These values of $q$ and $\eta$ correspond to the $p+{ }^{208} \mathrm{~Pb}$ system with $E_{c . m}=6 \mathrm{MeV}$. See Sects. 2 and 3 for discussion and definition of the relative error $\sigma_{k}$.

where

$$
\begin{aligned}
& \phi_{+}\left(\chi, q^{\prime}, l, \eta\right) \equiv u_{l, y}\left(q^{\prime}+\chi\right)\left(q^{\prime}+\chi\right)^{2} \mathcal{B}\left(\chi, q^{\prime}, l, \eta\right), \\
& \phi_{-}\left(\chi, q^{\prime}, l, \eta\right) \equiv u_{l, y}\left(q^{\prime}+\chi\right)\left(q^{\prime}+\chi\right)^{2} \mathcal{B}\left(\chi, q^{\prime}, l, \eta\right)^{*} .
\end{aligned}
$$

To apply the regularization scheme, we split the integrals $\mathcal{I}_{ \pm}$and take the limit $\pm i 0$,

$$
\mathcal{I}_{ \pm}=\int_{0}^{\Delta} d \chi \frac{\phi_{ \pm}\left(\chi, q^{\prime}, l, \eta\right)}{\chi^{1 \pm i \eta}}+e^{\pi \eta} \int_{0}^{\Delta} d \chi \frac{\phi_{ \pm}\left(-\chi, q^{\prime}, l, \eta\right)}{\chi^{1 \pm i \eta}} .
$$

While taking the limit, we used the following property,

$$
(\chi \pm i 0)^{-1 \mp i \eta}=|\chi|^{-1 \mp i \eta}\left(e^{ \pm i \pi}\right)^{-1 \mp i \eta}=-e^{\pi \eta}|\chi|^{-1 \mp i \eta} .
$$

By applying the Gel'fand-Shilov regularization technique from Ref. [4], we obtain

$$
\begin{aligned}
\mathcal{I}_{ \pm}=\int_{0}^{\Delta} d \chi \frac{\widetilde{\phi}_{ \pm}\left(\chi, q^{\prime}, l, \eta\right)}{\chi^{1 \pm i \eta}}+e^{\pi \eta} & \int_{0}^{\Delta} d \chi \frac{\widetilde{\phi}_{ \pm}\left(-\chi, q^{\prime}, l, \eta\right)}{\chi^{1 \pm i \eta}} \\
& \mp \frac{\phi_{ \pm}\left(0, q^{\prime}, l, \eta\right)}{i \eta}\left(1-e^{\pi \eta}\right) \Delta^{\mp i \eta}+\frac{\phi_{ \pm}^{\prime}\left(0, q^{\prime}, l, \eta\right)}{1 \mp i \eta}\left(1+e^{\pi \eta}\right) \Delta^{1 \mp i \eta},
\end{aligned}
$$

where

$$
\begin{aligned}
& \widetilde{\phi}_{ \pm}\left(\chi, q^{\prime}, l, \eta\right) \equiv \phi_{ \pm}\left(\chi, q^{\prime}, l, \eta\right)-\phi_{ \pm}\left(0, q^{\prime}, l, \eta\right)-\phi_{ \pm}^{\prime}\left(0, q^{\prime}, l, \eta\right) \chi \\
& \phi_{ \pm}^{\prime}\left(0, q^{\prime}, l, \eta\right)=\left.\frac{d \phi_{ \pm}\left(\chi, q^{\prime}, l, \eta\right)}{d \chi}\right|_{\chi=0}
\end{aligned}
$$

To calculate $\phi_{ \pm}^{\prime}\left(0, q^{\prime}, l, \eta\right)$, we also need $d u_{l, y}(p) / d p$. Taking the limit $\Delta \rightarrow+0$, one arrives at the expressions from Ref. [5].

By using the expressions from Eq. (11) and Eq. (6), we can compute the regularized part $I_{q-\Delta}^{q+\Delta}$ for any given finite $\Delta$. Then, using Eq. (5) we evaluate $u_{l, y}^{C}\left(q^{\prime}\right)^{\dagger}$, while $u_{l, z}^{C}(q)$ is given by

$$
u_{l, z}^{C}(q)=\left[\int \frac{d p p^{2}}{2 \pi^{2}} u_{l, z}(p)^{*} \psi_{l, q, \eta}^{C}(p)\right]^{*} .
$$


To calculate $\phi_{ \pm}^{\prime}\left(0, q^{\prime}, l, \eta\right)$, the expression for $d \mathcal{B} / d \chi$ is required. By using Eq. (B9) from Ref. [5], since $p=q^{\prime}+\chi$, Eq. (15.5.1) from Ref. [7], and by utilizing the property of the hypergeometric function ${ }_{2} F_{1}(\ldots ; 0)=1$, we obtain

$$
\left.\frac{d \mathcal{B}(\chi, q, l, \eta)}{d \chi}\right|_{\chi=0}=e^{-i \sigma_{l}} \Gamma(1+i \eta)(-3+i \eta) 2^{2 l-2+i \eta} q^{l-3+i \eta}
$$

Here we substituted $q^{\prime}$ by $q$ to simplify the expression.

\section{Results, Discussions and Outlook}

To prevent the unreasonably large computational times for calculating Coulomb distorted form factors, we improved the regularization scheme of Ref. [5]. This new scheme converges much faster, as the number of integration points increases. To demonstrate it, we present the error plot on Fig. 1. The integral $I_{a}^{b}$ (see caption for $a$ and $b$ ) was calculated by using Gauss-Legendre quadrature with 10, 20, $50,100,200,500,1000,2000$, and 5000 points. Starting from the second step ( $k=2$, i.e. 20 points), the relative discrepancy was calculated,

$$
\sigma_{k} \equiv\left(\frac{\left|\operatorname{Re}\left(I_{a}^{b}\right)_{k}-\operatorname{Re}\left(I_{a}^{b}\right)_{k-1}\right|}{\left|\operatorname{Re}\left(I_{a}^{b}\right)_{k}\right|}+\frac{\left|\operatorname{Im}\left(I_{a}^{b}\right)_{k}-\operatorname{Im}\left(I_{a}^{b}\right)_{k-1}\right|}{\left|\operatorname{Im}\left(I_{a}^{b}\right)_{k}\right|}\right) / 2,
$$

where $\left(I_{a}^{b}\right)_{k}$ is the value of $I_{a}^{b}$, obtained on the $k$-th step. For the sake of illustration, here we were using simple Yamaguchi-style real form factor with constant $2.7 \mathrm{fm}^{-1}$. It should be noted, that for this large value of $\eta=5.3$ and small value of $q=0.5 \mathrm{fm}^{-1}$ (the corresponding system is $p+{ }^{208} \mathrm{~Pb}$ at $E_{c . m .}=6 \mathrm{MeV}$ ) the final result is being calculated by subtraction of two large numbers, and the integral $I_{a}^{b}$ must be computed with $\sigma_{k} \lesssim 10^{-4}$ to get even the first digit of the final result. This new regularization scheme will be essential for successful progress in the numerical implementation of the Faddeev-AGS equations in Coulomb basis including spin degrees of freedom.

\section{Acknowledgements}

This work was performed in part under the auspices of the US Department of Energy, Office of Science of Nuclear Physics, under the topical collaborations in nuclear theory program No. DE-SC0004087 (TORUS Collaboration), and under Contract DE-FG02-93ER40756 with Ohio University.

\section{References}

[1] A. Deltuva, Phys. Rev. C 79, 054603 (2009).

[2] N. J. Upadhyay et al. Phys. Rev. C 85, 054621 (2012).

[3] A. M. Mukhamedzhanov et al. Phys. Rev. C 86, 034001 (2012).

[4] I. Gel'fand and G. Shilov, Generalized Functions, Volume 1: Properties and Operations (Academic, New York, 1964) Chapter 1, Sections 1.7, 2.2 (Example 3), 3.2, 3.8.

[5] N. J. Upadhyay et al. (the TORUS Collaboration), Phys. Rev. C 90, 014615 (2014).

[6] V. Eremenko et al. (the TORUS Collaboration), Comp. Phys. Comm. 187, 195 (2015).

[7] NIST Digital Library of Mathematical Functions (http://dlmf.nist.gov/, Release 1.0.10 of 2015-08-07, online companion to [8]).

[8] F. W. J. Olver, D. W. Lozier, R. F. Boisvert, and C. W. Clark, editors, NIST Handbook of Mathematical Functions (Cambridge University Press, New York, NY, 2010, Print companion to [7]). 